Pacific Journal of Mathematics

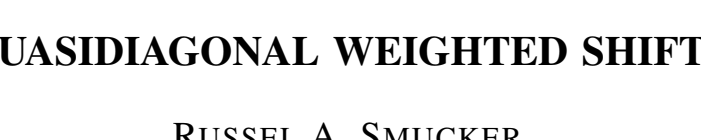




\title{
QUASIDIAGONAL WEIGHTED SHIFTS
}

\author{
RUSSELL SMUCKeR
}

We characterize the quasidiagonality of a two-way weighted shift solely in terms of the weights. We use the characterization to show that quasidiagonality fails to be an invariant for similarity: an operator similar to the (unweighted) bilateral shift may fail to be quasidiagonal.

Introduction. We given necessary and sufficient conditions for the quasidiagonality of a two-way weighted shift. We use the characterization to show that quasidiagonality fails to be invariant for similarity.

A (bounded) operator $A$ on a separable Hilbert space is quasidiagonal if there exists a sequence $\left\{P_{n}\right\}_{1}^{\infty}$ of orthogonal projections of finite rank such that $\left\{P_{n}\right\}$ converges strongly to 1 and $\left\{P_{n} A-A P_{n}\right\}$ converges uniformly to 0 . The only facts about quasidiagonal operators that we use in this paper are that each normal operator is quasidiagonal and that each compact perturbation of a quasidiagonal operator is again quasidiagonal. Both facts are proved in $[6, \S 4]$, where the concept was introduced.

Throughout this paper $\left\{e_{i}\right\}_{-\infty}^{+\infty}$ is a fixed basis for a complex Hilbert space, and $\left\{w_{i}\right\}_{-\infty}^{+\infty}$ is a fixed, bounded sequence of complex numbers. The operator $B$ determined on the Hilbert space by the equations $B e_{i}=w_{i} e_{i+1}$ is a two-way weighted shift with weight sequence $\left\{w_{i}\right\}_{-\infty}^{+\infty}$. The main goal of the paper is to prove Theorems 1,2 , and 5, which together characterize quasidiagonal weighted shifts solely in terms of their weight sequences.

\section{Sufficiency.}

THEOREM 1. If the weight sequence of a two-way weighted shift has 0 as a limit point in both directions, then it is quasidiagonal.

Proof. Let $\left\{w_{i_{n}}\right\}_{n=-\infty}^{+\infty}$ be a subsequence of the weights that converges to 0 in both directions. For each positive integer $n$, define $P_{n}$ to be the (orthogonal) projection onto the span of $\left\{e_{i_{-n}+1}, e_{i_{-n}+2}, \cdots\right.$, $\left.e_{i_{n}}\right\}$. It is easy to compute that $\left\|P_{n} B-B P_{n}\right\|$ is the larger of $\left|w_{i_{-n}}\right|$ and $\left|w_{i_{n}}\right|$; therefore $\left\|P_{n} B-B P_{n}\right\| \rightarrow 0$. The $P_{n}$ are increasing, and the union of their ranges contains all the vectors in the basis; therefore $P_{n} \rightarrow 1$ strongly. By definition, consequently, $B$ is quasidiagonal. 
Definition. A two-way sequence $\left\{w_{i}\right\}_{-\infty}^{+\infty}$ of complex numbers is block-balanced if for each positive number $\varepsilon$ and for each positive integer $n$ there exist integers $p$ and $q$ with $p+n<0<q$ and $\left|w_{p+k}-w_{q+k}\right|<\varepsilon$ for each $k$ with $0 \leqq k \leqq n$.

Roughly, $\left\{w_{i}\right\}_{-\infty}^{+\infty}$ is block-balanced if there exist arbitrarily long blocks (permitted to overlap) on each side of the center number that are arbitrarily near each other. It is obvious that the center number does not have to be at $w_{0}$; it could be at any fixed number $w_{i}$.

THEOREM 2. If the weight sequence of two-way weighted shift is block-balanced, then it is quasidiagonal.

We prove Theorem 2 after two lemmas. Next we describe a projection of the kind to be used in the proof. Let $n$ be a positive integer, and let $p$ and $q$ be integers with $p+n<0<q$. For each integer $k$ with $0 \leqq k \leqq n$, define vectors $g_{k}$ and $h_{k}$ by

$$
\begin{aligned}
& g_{k}=\sqrt{k / n} e_{p+k}+\sqrt{(n-k) / n} e_{q+k}, \\
& h_{k}=\sqrt{(n-k) / n} e_{p+k}-\sqrt{k / n} e_{q+k} .
\end{aligned}
$$

The set $\left\{g_{0}, g_{1}, \cdots, g_{n}, h_{0}, h_{1}, \cdots, h_{n}\right\}$ is an orthonormal set whose span is precisely the span of the orthonormal set $\left\{e_{p}, e_{p+1}, \cdots, e_{p+n}, e_{q}\right.$, $\left.e_{q+1}, \cdots, e_{q+n}\right\}$. (Note that there may be many vectors $e_{i}$ between $e_{p+n}$ and $e_{q}$.) We define the projection $P$, depending on $n, p$, and $q$, to be the projection onto the span of the orthonormal set $\left\{g_{0}, g_{1}, \cdots\right.$, $\left.g_{n}, e_{p+n+1}, e_{p+n+2}, \cdots, e_{q-1}\right\}$, (that is, the vectors $g_{k}$ and each vector $e_{i}$ between $e_{p+n}$ and $e_{q}$ ). The vectors $h_{k}$, though not involved directly in the definition of $P$, are nevertheless useful in the computations to come.

What is needed for Theorem 2 is an estimate for $\|P B-B P\|$; the next two lemmas provide it. Let $D$ be the diagonal operator (basis: $\left\{e_{i}\right\}_{-\infty}^{+\infty}$ ) with diagonal entries $\left\{w_{i}\right\}_{-\infty}^{+\infty}$, and let $W$ be the unweighted bilateral shift (same basis). Clearly, $B=W D$.

Lemma A. $\|P D-D P\| \leqq \max \left|w_{p+l}-w_{q+k}\right|$, (over $k$ with $\left.0 \leqq k \leqq n\right)$.

Proof. For each integer $k(0 \leqq k \leqq n)$ the space $M_{k}=\mathrm{V}\left\{g_{k}, h_{k}\right\}=$ $\bigvee\left\{e_{p+k}, e_{q+k}\right\}$ ("V" denotes span) reduces $P D-D P$, and $P D-D P$ vanishes on the orthogonal complement of the space spanned by all the vectors $g_{k}$ and all the vectors $h_{k}$. It is therefore sufficient to prove that

$$
\left\|(P D-D P)\left|M_{k} \| \leqq\right| w_{p+k}-w_{q+k} \mid \text { for } 0 \leqq k \leqq n .\right.
$$

Using the equations $P g_{k}=g_{k}$ and $P h_{k}=0$, one can easily com- 
pute that the matrix for $(P D-D P) \mid M_{k}$ with respect to the basis $\left\{g_{k}, h_{k}\right\}$ is the matrix

$$
\left(\begin{array}{cc}
0 & \left(D h_{k}, g_{k}\right) \\
-\left(D g_{k}, h_{k}\right) & 0
\end{array}\right)
$$

whose norm is the larger of $\left|\left(D g_{k}, h_{k}\right)\right|$ and $\left|\left(D h_{k}, g_{k}\right)\right|$. Now

$$
\begin{aligned}
\left|\left(D g_{k}, h_{k}\right)\right|= & \mid\left(w_{p+k} \sqrt{k / n} e_{p+k}+w_{q+k} \sqrt{(n-k) / n} e_{q+k},\right. \\
& \left.\sqrt{(n-k) / n} e_{p+k}-\sqrt{k / n} e_{q+k}\right) \mid \\
= & \sqrt{k(n-k)} / n\left|w_{p+k}-w_{q+k}\right| \leqq\left|w_{p+k}-w_{q+k}\right| .
\end{aligned}
$$

A similar computation shows that $\left|\left(D h_{k}, g_{k}\right)\right| \leqq\left|w_{p+k}-w_{q+k}\right|$; therefore $\left\|(P D-D P)\left|M_{k} \| \leqq\right| w_{p+k}-w_{q+k} \mid\right.$.

\section{Lemma B. $\|P W-W P\| \leqq 2 / \sqrt{n}$.}

Proof. The basic idea of the proof is quite simple: each vector in the orthonormal set $\left\{g_{0}, \cdots, g_{n}, e_{p+n+1}, \cdots, e_{q-1}\right\}$ is mapped by $W$ almost into the next one (and the last vector is mapped almost into the first one). It makes sense therefore that the span of the set, which is by definition the range of $P$, should be almost reducing. The computations are simplified if we note that $\|P W-W P\| \leqq$ $\|(1-P) W P\|+\|P W(1-P)\|$; we shall show that each of the two summands on the right is bounded above by $1 / \sqrt{n}$.

To estimate $\|(1-P) W P\|$, begin by computing $(1-P) W f$ for each basis vector $f$ in the orthonormal basis $\left\{g_{0}, \cdots, g_{n}, e_{p+n+1}, \cdots, e_{q-1}\right\}$ for the range of $P$. For each integer $i$ with $p+n+1 \leqq i \leqq q-1$, the vector $e_{i+1}$ is in the range of $P$ (note that $e_{q}=g_{0}$ ); therefore $(1-P) W e_{i}=(1-P) e_{i+1}=0$. For each integer $k$ with $0 \leqq k \leqq n-1$, the vector $W g_{k}$ is in $\mathrm{V}\left\{e_{p+k+1}, e_{q+k+1}\right\}=\mathrm{V}\left\{g_{k+1}, h_{k+1}\right\}$. Because the vector $g_{k+1}$ is in the range of $P$, and because the vector $h_{k+1}$ is in the range of $1-P$, it follows that $(1-P) W g_{k}=\left(W g_{k}, h_{k+1}\right) h_{k+1}$. Finally, $g_{n}=e_{p+n}$; therefore $(1-P) W g_{n}=(1-P) e_{p+n+1}=0$. The computations so far show that $(1-P) W$ annihilates all the vectors in the basis for the range of $P$ except $g_{0}, \cdots, g_{n-1}$, each of which it maps into a multiple of the corresponding vector in the orthonormal set $\left\{h_{1}, \cdots, h_{n}\right\}$. The norm of $(1-P) W P$ is therefore simply the largest absolute value of the multiples; that is, $\|(1-P) W P\|=$ $\max \left|\left(W g_{k}, h_{k+1}\right)\right|,(0 \leqq k \leqq n-1)$. Now,

$$
\left|\left(W g_{k}, h_{k+1}\right)\right|=|\sqrt{k(n-k-1)}-\sqrt{(n-k)(k+1)}| / n \leqq 1 / \sqrt{n} .
$$

This proves that $\|(1-P) W P\| \leqq 1 / \sqrt{n}$. 
The estimation of $\|P W(1-P)\|$ is similar to that for $\|(1-P) W P\|$; therefore we give only a sketch. The vectors in the basis $\left\{\cdots, e_{p-2}\right.$, $\left.e_{p-1}, h_{0}, \cdots, h_{n}, e_{q+n+1}, e_{q+n+2}, \cdots\right\}$ for the range of $1-P$ are all annihilated by $P W$ except $h_{0}, \cdots, h_{n-1}$, and $P W h_{k}=\left(W h_{k}, g_{k+1}\right) g_{k+1}$ for $0 \leqq k \leqq n-1$. Therefore $\|P W(1-P)\| \leqq \max \left|\left(W h_{k}, g_{k+1}\right)\right|, \quad(0 \leqq$ $k \leqq n-1)$, and the right side is again bounded by $1 / \sqrt{n}$.

Proof of Theorem 2. Since the weight sequence $\left\{w_{i}\right\}_{-\infty}^{+\infty}$ is blockbalanced, there exists for each positive integer $n$ a pair of integers $p$ and $q$ such that $p+n<0<q$ and $\left|w_{p+k}-w_{q+k}\right|<1 / n$ for $0 \leqq k \leqq n$. Define $P_{n}$ to be the projection $P$ (depending on $n, p$, and $q$ ) defined just before Lemma $A$. We shall prove that the sequence $\left\{P_{n}\right\}_{1}^{+\infty}$ of projections of finite rank implements the quasidiagonality of $B=W D$.

Because $P_{n} B-B P_{n}=\left(P_{n} W-W P_{n}\right) D+W\left(P_{n} D-D P_{n}\right)$, it follows that

$$
\left\|P_{n} B-B P_{n}\right\| \leqq\left\|P_{n} W-W P_{n}\right\|\|D\|+\|W\|\left\|P_{n} D-D P_{n}\right\| .
$$

By the two lemmas, the right side is dominated by $2\|D\| / \sqrt{n}+$ $\|W\| / n$, so that $\left\|P_{n} B-B P_{n}\right\| \rightarrow 0$.

It remains to show that $P_{n} \rightarrow 1$ strongly; it is sufficient to prove that $P_{n} e_{i} \rightarrow e_{i}$ as $n \rightarrow \infty$, for each basis vector $e_{i}$. Two cases must be considered: $i<0$ and $i \geqq 0$. The proofs for the two cases differ in notation only; therefore we prove only the case $i \geqq 0$. Also, we need to consider only $n \geqq i$. If the $q$ corresponding to $n$ satisfies the inequality $q>i$, then $p+n+1 \leqq 0 \leqq i \leqq q-1$, so that $e_{i}$ is in the range of $P_{n}$, and $P_{n} e_{i}=e_{i}$. If on the other hand, $q \leqq i$, let $k=i-q$, and note that $0 \leqq k \leqq n$. The basis vector $e_{i}=e_{q+k}$ is in $\bigvee\left\{e_{q+k}, e_{p+k}\right\}=$ V $\left\{g_{k}, h_{k}\right\} ;$ consequently $P_{n} e_{i}=\left(e_{q+k}, g_{k}\right) g_{k}=\sqrt{(n-k) k} / n e_{p+k}+(n-$ $k) / n e_{q+k}$. It follows that $P_{n} e_{i}-e_{i}=\sqrt{(n-k) k} / n e_{p+k}-k / n e_{q+k}$, so that $\left\|P_{n} e_{i}-e_{i}\right\|^{2}=k / n$. The condition $0<q$ implies that $k=i-q<i$, so that $\left\|P_{n} e_{i}-e_{i}\right\|^{2}<i / n$. In summary, we have proved in either case $(q>i$ or $q \leqq i)$ that $\left\|P_{n} e_{i}-e_{i}\right\|^{2}<i / n$, provided $n \geqq i$. For each integer $i \geqq 0$, therefore, $P_{n} e_{i} \rightarrow e_{i}$ as $n \rightarrow \infty$.

COROLLARY. If the weights of a two-way weighted shift are periodic, then it is quasidiagonal.

Proof. If the weights are periodic, then they are blockbalanced.

In case the weights are strictly positive, it is interesting to compare the corollary with a result of $R$. L. Kelley, which says that $B$ is reducible if and only if the weights are periodic [7, Thm. 11, 
p. 44]. The proof of Kelley's result produces projections that commute with $B$ but that are (unavoidably) of infinite rank. The proof of the corollary produces projections that are of finite rank and that nearly commute with $B$, but that cannot actually commute with $B$.

2. Necessity. The theorems in $\S 1$ produce two kinds of quasidiagonal two-way weighted shifts. In this section, we prove that they are the only ones. The key idea is that for each shift that does not belong to one of the two kinds, there is a nonquasitriangular operator in the $C^{*}$-algebra generated by the shift, so that by Theorem 3 the shift itself fails to be quasidiagonal.

Throughout this section, it is necessary to assume that the weights are nonnegative (Theorems 4 and 5 are false, otherwise), and it is convenient to assume that $\|B\| \leqq 1$ (so that $0 \leqq w_{i} \leqq 1$ ). No real generality is lost in either case, for the weighted shift with weights $\left\{w_{i}\right\}_{-\infty}^{+\infty}$ is unitarily equivalent to the weighted shift with weights $\left\{\left|w_{i}\right|\right\}_{-\infty}^{+\infty}[4$, Problem 75], and quasidiagonality is unchanged by multiplication by scalars (this is obvious from the definition).

THEOREM 3. If $A$ is quasidiagonanl, then each operator in the $C^{*}$-algebra generated by $A$ is also quasidiagonal.

Proof. If the sequence $\left\{P_{n}\right\}$ of projections of finite rank implements the quasidiagonality of $A$, then $\left\{P_{n}\right\}$ also implements the quasidiagonality of $A^{*}$, because $\left\|P_{n} A^{*}-A^{*} P_{n}\right\|=\left\|P_{n} A-A P_{n}\right\|$. It therefore implements the quasidiagonality of finite products involving $A$ and $A^{*}$, because $\left\|P_{n} S T-S T P_{n}\right\| \leqq\left\|P_{n} S-S P_{n}\right\|\|T\|+\|S\| \| P_{n} T-$ $T P_{n} \|$; also it implements all polynomials (possibly noncommutative) in $A$ and $A^{*}$, because

$$
\left\|P_{n}(S+T)-(S+T) P_{n}\right\| \leqq\left\|P_{n} S-S P_{n}\right\|+\left\|P_{n} T-T P_{n}\right\| .
$$

If, finally, $S_{n} \rightarrow S$ and each $S_{n}$ is a polynomial in $A$ and $A^{*}$, then by a diagonal argument it is possible to select a subsequence $\left\{P_{n_{k}}\right\}$ of $\left\{P_{n}\right\}$ that implements the quasidiagonality of $S$; the inequality needed is

$$
\begin{aligned}
\left\|P_{n_{k}} S-S P_{n_{k}}\right\| \leqq & \left\|P_{n_{k}}\right\|\left\|S-S_{n}\right\|+\left\|S_{n}-S\right\|\left\|P_{n_{k}}\right\| \\
& +\left\|P_{n_{k}} S_{n}-S_{n} P_{n_{k}}\right\| .
\end{aligned}
$$

The next theorem provides the tool necessary for applying Theorem 3 in the context of weighted shifts. In its proof, we need an approximation theorem of the Stone-Weierstrass type. Let $n$ be a fixed, nonnegative integer and let $X$ be the Cartesian product of 
$n+1$ copies of the closed unit interval $[0,1]$, the component functions being indexed by the integers 0 to $n$ (so that a typical point in $X$ is $\left(x_{0}, x_{1}, \cdots, x_{n}\right)$ with $\left.0 \leqq x_{i} \leqq 1\right)$. Let $C^{r}(X)$ be the normed space of real-valued continuous functions on $X$ with the sup norm. We need the fact that if the functions in a subset $S$ of $C^{r}(X)$ vanish identically on a closed subset $X_{0}$ of $X$ and separate points in the complement of $X_{0}$, then the algebra of functions generated by $S$ is dense in the set $C_{0}$ of functions in $C^{r}(X)$ that vanish identically on $X_{0}[9$, Thm. 7, p. 46].

THEOREM 4. If $u$ is a function in $C^{r}(X)$ that vanishes identically on the coordinate faces $x_{j}=0(0 \leqq j \leqq n)$, then the weighted shift $B_{u}$ with weights $\left\{u\left(w_{i}, w_{i+1}, \cdots, w_{i+n}\right)\right\}_{i=-\infty}^{+\infty}$ is in the $C^{*}$-algebra generated by the weighted shift $B$ with weights $\left\{w_{i}\right\}_{-\infty}^{-\infty}\left(0 \leqq w_{i} \leqq 1\right)$.

Proof. Let $\mathscr{A}[B]$ be the $C^{*}$-algebra generated by $B$. If $j$ is a nonnegative integer, then the operator $D_{j}$ defined by the formula $D_{j}=\sqrt{B^{*(j+1)} B^{(j+1)}}$ is the diagonal operator with diagonal entries $\left\{w_{i} w_{i+1} \cdots w_{i+j}\right\}_{i=-\infty}^{+\infty}$ (the assumption $0 \leqq w_{i}$ has just been used), and $D_{j}$ is in $\mathscr{A}[B]$ (because $D_{j}$ is in $\mathscr{A}\left[D_{j}^{2}\right]$ by the Gelfand-Naìmark theorem [3, Thm. 7, p. 876], and $\left.\mathscr{A}\left[D_{j}^{2}\right] \subset \mathscr{A}[B]\right)$. The product $B D_{j}$ is the weighted shift with weights $\left\{w_{1}^{2} w_{i+1} w_{i+2} \cdots w_{i+j}\right\}_{i=-\infty}^{+\infty}$, and it is in $\mathscr{A}[B]$. More generally, if $b_{0}, b_{1}, \cdots, b_{n}$ is a sequence of nonnegative integers, then the operator $B D_{0}^{b_{0}} D_{1}^{b_{1}} D_{2}^{b_{2}} \cdots D_{n}^{b_{n}}$ is the weighted shift whose $i$ th weight is

$$
w_{i}^{\left(1+b_{0}+b_{1}+\cdots+b_{n}\right)} w_{i+1}^{\left(b_{1}+b_{2}+\cdots+b_{n}\right)} w_{i+2}^{\left(b_{2}+b_{3}+\cdots+b_{n}\right)} \cdots w_{i+n}^{b_{n}},
$$

and it is in $\mathscr{C}[B]$. Put another way, if $m_{0}, m_{1}, \cdots, m_{n}$ is a sequence of integers with $m_{0} \geqq m_{1} \geqq \cdots \geqq m_{n} \geqq 0$, and if $u$ is the function in $C^{r}(X)$ defined by $u\left(x_{0}, \cdots, x_{n}\right)=x_{3}^{\left(m_{0}+1\right)} x_{1}^{m_{1}} x_{2}^{m_{2}} \cdots x_{n}^{m_{n}}$, then the weighted shift $B_{u}$ is in $\mathscr{A}[B]$.

Let $C_{0}$ be the subset of $C^{r}(X)$ consisting of the functions in $C^{r}(X)$ that vanish on the set $X_{0}$ of points in $X$ one of whose coordinates is 0 . We want to show that $B_{u}$ is in $\mathscr{A}[B]$ whenever $u$ is in $C_{0}$; the first paragraph proves that $B_{u}$ is in $\mathscr{A}[B]$ whenever $u$ is in the set $S$ consisting of the functions in $C^{r}(X)$ that have the special form $u\left(x_{0}, \cdots, x_{n}\right)=x_{0}^{\left(m_{0}+1\right)} x_{1}^{m_{1}} x_{2}^{m_{2}} \cdots x_{n}^{m_{n}}$, where the $m_{i}$ are integers such that $m_{0} \geqq m_{1} \geqq \cdots \geqq m_{n} \geqq 0$. Because the set $S$ is closed under multiplication, each function in the algebra $\mathscr{S}$ generated by $S$ is merely a linear combination of functions in $S$. Because $B_{u_{1}}+$ $B_{u_{2}}=B_{u_{1}+u_{2}}$ and $B_{\alpha u}=\alpha B_{u}$ for each scalar $\alpha$, the operator $B_{u}$ is in $\mathscr{A}[B]$ whenever $u$ is in $\mathscr{S}$. If $u_{n} \rightarrow u$ uniformly on $X$, then $B_{u_{n}} \rightarrow B_{u}$ uniformly; therefore $B_{u}$ is in $\mathscr{A}[B]$ whenever $u$ is in the uniform closure of $\mathscr{S}$. The set $S$ separates points in $X-X_{0}$; therefore, by 
the remark preceding the theorem, the uniform closure of $\mathscr{S}$ is all of $C_{0}$.

The condition $0 \leqq w_{i}$ in Theorem 4 is necessary. To see this, suppose the weights of $B$ are

$$
\{\cdots,-1,1,-1,(1),-1,-1,1,-1,-1, \cdots\}
$$

(note that the weight 1 alternates with a single -1 in the backward direction while it alternates with a pair of -1 's in the forward direction). The operator $B$ is quasidiagonal (because it is normal); therefore each operator in $\mathscr{A}[B]$ is quasidiagonal, by Theorem 3 . If $u$ is a continuous function of one variable such that $u(1)=1$ and $u(-1)=2$, then $B_{u}$ has weights $\{\cdots, 2,1,2,(1), 2,2,1,2,2, \cdots\}$, and $B_{u}$ fails to be quasidiagonal (as the next theorem shows); therefore $B_{u}$ is not in $\mathscr{C}[B]$.

THEOREM 5. If a two-way weighted shift with nonnegative weights is quasidiagonal, then either the weight sequence has 0 as a limit point in both directions, or it is block-balanced.

Proof. Suppose that the weight sequence does not have 0 has a limit point in one direction and that it is not block-balanced. We must prove that $B$ is not quasidiagonal. Since quasidiagonality is invariant under the adjoint operation, it is sufficient to prove the case where the weight sequence does not have 0 as a limit point in the forward direction. It is then possible to alter a finite number of the weights to produce a new weight sequence (which is still not block-balanced) that is bounded below in the forward direction by a positive number, say $\delta$. Such a change amounts to a compact perturbation of $B$, and as such does not alter quasidiagonality.

The assumptions now are that $0 \leqq w_{i} \leqq 1$ for each integer $i$, that $0<\delta \leqq w_{i} \leqq 1$ for $i \geqq 0$, and that there exists a positive number $\varepsilon$ and a positive integer $n$ such that to each pair of integers $p$ and $q$ with $p+n<0<q$ there corresponds an integer $k(0 \leqq k \leqq n)$ such that $\left|w_{p+k}-w_{q+k}\right| \geqq \varepsilon$. We must prove that $B$ is not quasidiagonal. Let $Y$ be the closure in the normed space $X$ (defined just before Theorem 4$)$ of the set of points $\left\{\left(w_{i}, w_{i+1}, \cdots, w_{i+n}\right)\right\}_{i=0}^{+\infty}$ (note: in the forward direction only), and let $Z$ be the closure of the set consisting first of the points in $\left\{\left(w_{i}, w_{i+1}, \cdots, w_{i+n}\right)\right\}_{i=-\infty}^{n}$ (note: in the backward direction only) and second of the points in $X$ at least one of whose components is 0 . The assumption on $\varepsilon$ and $n$ implies that the set $Y$ is at a distance at least $\varepsilon$ from each point of the first kind in $Z$, and the assumption on $\delta$ implies that $Y$ is at a distance 
at least $\delta$ from each point of the second kind in $Z$. The closed sets $Y$ and $Z$ are therefore disjoint. By Urysohn's lemma, there exists a continuous function $u$ on $X$ with $u[Z]=0$ and $u[Y]=1$. The function $u$ vanishes on the face $x_{0}=0$ of Theorem 4 because the points in that face are contained in $Z$.

By Theorem 4 , the shift $B_{u}$ is in the $C^{*}$-algebra generated by $B$. If $B$ were quasidiagonal, then by Theorem $3, B_{u}$ would also be quasidiagonal. Except for a compact perturbation, $B_{u}$ is the weighted shift with weights $\{\cdots, 0,0,0,(1), 1,1, \cdots\}$ (the weights with indices from $-n+1$ to -1 may be different). The quasidiagonality of $B$ thus implies the quasidiagonality of $0 \oplus U$ ( $U$ is the unweighted unilateral shift). It is known, however, that $0 \oplus U$ fails even to be quasitriangular (proof: $U$ is not quasitriangular [5, Thm. 3]; therefore neither is $0 \oplus U$ [2, Thm. 8]). The weighted shift $B$ consequently fails to be quasidiagonal.

3. Applications. In this section we give two applications that use the characterization of quasidiagonal weighted shifts developed in the previous two sections. The first application is a reformulation of the characterization in a special case, namely where the weighted shift has compact self-commutator (the self-commutator of $A$ is $\left.A^{*} A-A A^{*}\right)$. The second example shows that quasidiagonality fails to be invariant for similarity.

THEOREM 6. A two-way weighted shift with nonnegative weights that has compact self-commutator is quasidiagonal if and only if there exists a number that is both a forward limit point and a backward limit point of the weight sequence.

L. G. Brown, R. G. Douglas, and P. A. Fillmore have proved that if an operator $A$ with compact self-commutator is quasidiagonal, then it is a compact perturbation of a diagonal operator [1, Cor. 11.12]. Theorem 6 therefore also characterizes two-way weighted shifts that are compact perturbations of diagonal operators.

Proof. A diagonal operator with weights $\left\{d_{i}\right\}_{-\infty}^{+\infty}$ is compact if and only if the weights converge to 0 in both directions. Since $B^{*} B-B B^{*}$ is the diagonal operator with weights $\left\{w_{i}^{2}-w_{i+1}^{2}\right\}_{-\infty}^{+\infty}$, it follows that $B^{*} B-B B^{*}$ is compact if and only if $\left\{w_{i}-w_{i+1}\right\}_{-\infty}^{+\infty}$ converges to 0 in both directions. In that case, the set of forward limit points is a closed interval $R$, and the set of backward limit points of $\left\{w_{i}\right\}_{-\infty}^{+\infty}$ is a closed interval $L$. 
If $R$ and $L$ have a point in common, say $x$, then, because $\left\{w_{i}-w_{i+1}\right\}_{-\infty}^{+\infty}$ converges to 0 in both directions, there exist arbitrarily long blocks, on both sides of the center weight, such that the weights in the blocks are arbitrarily near to $x$, and therefore arbitrarily near to each other. The weight sequence is therefore block-balanced, so that $B$ is quasidiagonal by Theorem 2 .

If $R$ and $L$ are disjoint, then they are a positive distance apart. The condition that $\left\{w_{i}-w_{i+1}\right\}_{-\infty}^{+\infty}$ converges to 0 in both directions means that eventually the blocks to the right of the center weight are a positive distance (sup norm) from the blocks to the left of the center weight. The weight sequence is therefore not block-balanced. Neither does it have 0 as a limit point in both directions, since $R$ and $L$ are disjoint. By Theorem 5 , the shift $B$ fails to be quasidiagonal.

EXAMPLE. An operator that is similar to the (unweighted) bilateral shift $W$ may fail to be quasidiagonal.

Proof. Let $A$ be the two-way weighted shift with weights $\{\cdots, 1,1,1,(1 / 2), 2,1 / 2,2, \cdots\}$. If $S$ is the diagonal operator with diagonal entries $\{\cdots, 1,1,1,(1), 2,1,2, \cdots\}$, then $S^{-1} A S=W$, so that $A$ is similar to $W$. By Theorem 5, on the other hand, $A$ fails to be quasidiagonal.

\section{REFERENCES}

1. L. G. Brown, R. G. Douglas and P. A. Fillmore, Unitary equivalence modulo the compact operators and extensions of $C^{*}$-algebras, Procedings of a Conference on Operator Theory, P. A. Fillmore, ed., Lecture Notes in Mathematics, Volume 345, Springer-Verlag, New York, 1973.

2. R. G. Douglas and C. Pearcy, A note on quasitriangular operators, Duke Math. J., 37 (1970), 177-188.

3. N. Dunford and J.T. Schwartz, Linear Operators, Part II: Spectral Theory, Pure and Applied Mathematics, Vol. VII, Interscience, New York, 1963.

4. P. R. Halmos, A Hilbert space problem book, The university series in higher mathematics, D. Van Nostrand, Princeton, 1967.

5. - - Quasitriangular operators, Acta Sci. Math., (Szeged) 29 (1968), 283-293.

6. - Ten problems in Hilbert space, Bull. Amer. Math. Soc., 76 (1970), 877933.

7. R. L. Kelley, Weighted shifts on Hilbert space, Dissertation, University of Michigan, Ann Arbor, Mich., 1966.

8. R. A. Smucker, Quasidiagonal and quasitriangular operators, Dissertation, Indiana University, Bloomington, Ind., 1973.

9. M. H. Stone, A generalized Weierstrass approximation theorem, Studies in modern analysis, R. C. Buck, ed., Studies in mathematics, Vol. 1, Prentice-Hall, Englewood Cliffs, N. J., 1962.

Received May 1, 1979. This paper is part of the author's doctoral thesis at Indiana University under the direction of P. R. Halmos. 
Kalamazoo College KALAMAZOO, MI 49007 AND

UNIVERSITY OF ZAMBIA

Box 2379

LUSAKa, ZAMBIA 


\title{
PACIFIC JOURNAL OF MATHEMATICS
}

\section{EDITORS}

\author{
DONALD BABBITT (Managing Editor) \\ University of California \\ Los Angeles, CA 90024 \\ Hugo RossI \\ University of Utah \\ Salt Lake City, UT 84112 \\ C. C. Moore and ANDrew OGG \\ University of California \\ Berkeley, CA 94720
}

\section{J. DugundjI}

Department of Mathematics

University of Southern California

Los Angeles, CA 90007

R. FinN and J. Milgram

Stanford University

Stanford, CA 94305

\section{ASSOCIATE EDITORS}
R. ARENS
E. F. BeCKENBACH
B. H. NeUMANN
F. WOLF
K. YoshidA

\section{SUPPORTING INSTITUTIONS}

UNIVERSITY OF ARIZONA

UNIVERSITY OF BRITISH COLUMBIA

CALIFORNIA INSTITUTE OF TECHNOLOGY

UNIVERSITY OF CALIFORNIA

MONTANA STATE UNIVERSITY

UNIVERSITY OF NEVADA, RENO

NEW MEXICO STATE UNIVERSITY

OREGON STATE UNIVERSITY

\author{
UNIVERSITY OF OREGON \\ UNIVERSITY OF SOUTHERN CALIFORNIA \\ STANFORD UNIVERSITY \\ UNIVERSITY OF HAWAII \\ UNIVERSITY OF TOKYO \\ UNIVERSITY OF UTAH \\ WASHINGTON STATE UNIVERSITY \\ UNIVERSITY OF WASHINGTON
}

The Supporting Institutions listed above contribute to the cost of publication of this Journal, but they are not owners or publishers and have no responsibility for its content or policies.

Mathematical papers intended for publication in the Pacific Journal of Mathematics should be in typed form or offset-reproduced, (not dittoed), double spaced with large margins. Please do not use built up fractions in the text of the manuscript. However, you may use them in the displayed equations. Underline Greek letters in red, German in green, and script in blue. The first paragraph or two must be capable of being used separately as a synopsis of the entire paper. Please propose a heading for the odd numbered pages of less than 35 characters. Manuscripts, in triplicate, may be sent to any one of the editors. Please classify according to the scheme of Math. Reviews, Index to Vol. 39. Supply name and address of author to whom proofs should be sent. All other communications should be addressed to the managing editor, or Elaine Barth, University of California, Los Angeles, California, 90024.

50 reprints to each author are provided free for each article, only if page charges have been substantially paid. Additional copies may be obtained at cost in multiples of 50 .

The Pacific Journal of Mathematics is issued monthly as of January 1966. Regular subscription rate: $\$ 102.00$ a year (6 Vols., 12 issues). Special rate: $\$ 51.00$ a year to individual members of supporting institutions.

Subscriptions, orders for numbers issued in the last three calendar years, and changes of address shoud be sent to Pacific Journal of Mathematics, P.O. Box 969, Carmel Valley, CA 93924, U.S.A. Old back numbers obtainable from Kraus Periodicals Co., Route 100, Millwood, NY 10546.

PUBLISHED BY PACIFIC JOURNAL OF MATHEMATICS, A NON-PROFIT CORPORATION

Printed at Kokusai Bunken Insatsusha (International Academic Printing Co., Ltd.). 8-8, 3-chome, Takadanobaba, Shinjuku-ku, Tokyo 160, Japan. 


\section{Pacific Journal of Mathematics}

\section{Vol. 98, No. 1 \\ March, 1982}

Humberto Raul Alagia, Cartan subalgebras of Banach-Lie algebras of

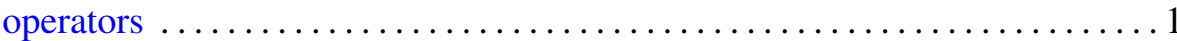

Tom M. (Mike) Apostol and Thiennu H. Vu, Elementary proofs of

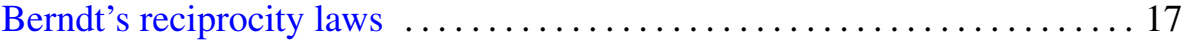

James Robert Boone, A note on linearly ordered net spaces $\ldots \ldots \ldots \ldots . \ldots 25$

Miriam Cohen, A Morita context related to finite automorphism groups of

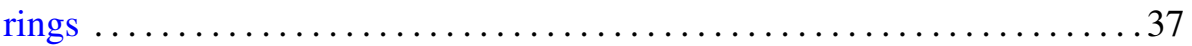

Willibald Doeringer, Exceptional values of differential polynomials . . . . . . 55

Alan Stewart Dow and Ortwin Joachim Martin Forster, Absolute

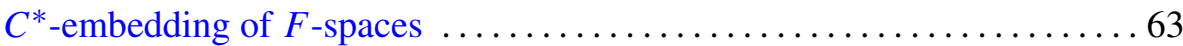

Patrick Hudson Flinn, A characterization of $M$-ideals in $B\left(l_{p}\right)$ for

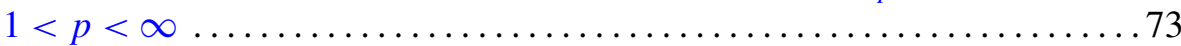

Jack Emile Girolo, Approximating compact sets in normed linear spaces . . . 81

Antonio Granata, A geometric characterization of $n$th order convex

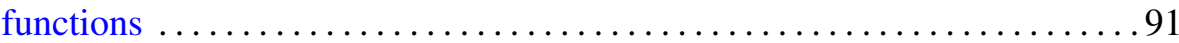

Kenneth Richard Johnson, A reciprocity law for Ramanujan sums . . . . . . .99

Grigori Abramovich Kolesnik, On the order of $\zeta\left(\frac{1}{2}+i t\right)$ and $\Delta(R) \ldots \ldots 107$

Daniel Joseph Madden and William Yslas Vélez, Polynomials that

represent quadratic residues at primitive roots $\ldots \ldots \ldots \ldots \ldots \ldots \ldots 123$

Ernest A. Michael, On maps related to $\sigma$-locally finite and $\sigma$-discrete

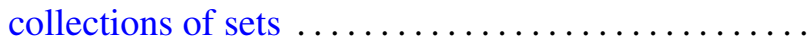

Jean-Pierre Rosay, Un exemple d'ouvert borné de $\mathbf{C}^{3}$ “taut" mais non

hyperbolique complet

Roger Sherwood Schlafly, Universal connections: the local problem

Russel A. Smucker, Quasidiagonal weighted shifts .....

Eduardo Daniel Sontag, Remarks on piecewise-linear algebra . .

Jan Søreng, Symmetric shift registers. II

H. M. (Hari Mohan) Srivastava, Some biorthogonal polynomials suggested

by the Laguerre polynomials 